\section{Hippocampal electrical activity during hypothalamic-evoked consummatory behavior in rats*}

\author{
FRED J. POND,$\dagger$ THEODORE I. LIDSKY, MICHAEL S. LEVINE, $\nmid \dagger$ \\ and J. S. SCHWARTZBAUM \\ University of Rochester, Rochester, N.Y. 14627
}

Hippocampal electrical activity was recorded in freely moving animals during normal feeding behavior, lateral-hypothalamic stimulation, and during eating and drinking induced by such stimulation. In contrast to the irregular and desynchronized hippocampal activity observed during normal feeding behavior, stimulus-bound eating and drinking were associated with synchronization of hippocampal activity. The peak frequency of theta rhythms during such elicited consummatory behavior was $1.2 \mathrm{~Hz}$ below that evoked by the stimulation in the absence of consummatory objects. The results point up differences between normal and stimulus-evoked consummatory behavior and the influence of consummatory activity upon electrophysiological state.

Positively rewarding stimulation of the lateral hypothalamus (LH) has been shown to evoke synchronization of hippocampal EEG activity with a peak frequency of about $8-10 \mathrm{~Hz}$, depending upon the intensity of the stimulation (Kramis \& Routtenberg, 1969 ; Pond \& Schwartzbaum, 1970). However, the significance of this evoked hippocampal "theta" pattern remains unclear. It does not relate to the positive rewarding properties of the stimulation as suggested by Grastyan and his coworkers (Grastyan, Karmos, Vereczkey, \& Kellenyi, 1966). Aversive stimulation of the dorsomedial tegementum of the midbrain and medial hypothalamus, which evokes escape behavior but not self-stimulation, also elicits 8- to $10-\mathrm{Hz}$ hippocampal theta activity (Pond \& Schwartzbaum, 1970; Rout tenberg \& Kramis, 1968); and stimulation of the septal area, which typically supports self-stimulation, induces desynchronization rather than synchronization of hippocampal EEG (Pond \& Schwartzbaum, 1970). Similarly, there are grounds for rejecting the supposition (Pond \& Schwartzbaum, 1970) of a relationship between evoked high-frequency or fast theta (above $8 \mathrm{~Hz}$ in the rat) and aversive properties of brain stimulation, i.e., in terms of suppressing subsequent behavior initiating the stimulation.

We have suggested the possibility that hippocampal theta patterns produced by rewarding brain stimulation might relate to some parameter of appetitive activity

* This study was supported by Grant MH-14594 from the National Institute of Mental Health. We wish to thank Miss Gertrude Lusk for her capable histological work.

+ Traince supported by Grant $\mathrm{MH}-10825$.

$\dagger+N S F$ predoctoral fellow. evoked by the stimulation (Pond \& Schwartzbaum, 1970). LH stimulation, e.g., typically elicits exploratory behavior (Christopher \& Butter, 1968) and there is, indeed, a close relationship between stimulus thresholds for evoking hippocampal theta activity and exploratory patterns (Pond \& Schwartzbaum, 1970). A similar relationship exists between theta activity and normally occurring investigatory behavior (Routtenberg, 1968; Vanderwolf, 1969), although the latter is not a necessary condition (Vanderwolf, 1969). Under other conditions, LH stimulation may give rise to conditionable sequences of response directed toward consummatory activities or species-specific patterns of behavior such as eating, drinking, copulation, gnawing, etc. (Valenstein, Cox, \& Kakolewski, 1969). Relatively little is known about the hippocampal EEG correlates of such evoked patterns that involve appetitive and consummatory components. Normally, consummatory and species-specific modes of behavior are associated with irregularity, low frequency, or desynchronization of hippocampal activity (Routtenberg, 1968; Vanderwolf, 1969). Appetitive sequences leading to consummatory activity are typically associated with some shift in hippocampal state toward such patterns of activity. The present experiment provides data on the hippocampal correlates of stimulus-bound eating and drinking and points up electrophysiological differences from corresponding patterns of behavior that are normally motivated.

\section{METHOD}

Three male Holtzman rats, weighing approximately $350 \mathrm{~g}$, that displayed stimulus-bound consummatory behavior served as Ss. Using standard surgical procedures, each animal was implanted stereotactically with bipolar recordingleads in each dorsal hippocampus. The leads were made from insulated .008-in. stainless steel wires, twisted together with only the cross-sectional tips left uninsulated. A monopolar stimulating electrode (No. 0 stainless steel insect pin, insulated except for $0.5 \mathrm{~mm}$ at the tip) was implanted in the lateral hypothalamus unilaterally. The circuit was completed by a stainless steel screw implanted over the frontal cortex. An additional frontal screw served as a ground connection. Coordinates for the hippocampal and hypothalamic electrodes are available elsewhere (Pond \& Schwartzbaum, 1970). Each lead was crimped to an Amphenol pin (220PO2) that was inserted into an Amphenol plug strip (221-2253). The electrode assembly was secured to the skull by means of acrylic cement and stainless steel screws.

Observations on electrophysiological activity, self-stimulation, and evoked behavior were obtained in a small test chamber containing retractable bars (Lehigh Valley 1405R) that was housed in a IAC enclosure. The electrode plug was attached to a Microdot cable assembly that linked to a Lehigh Valley swivel (1373-9). Electrical stimulation (100 pulses/sec of alternating polarity, each pulse $0.3 \mathrm{msec}$ in duration) was controlled by a Nuclear-Chicago constant-current stimulator. Hippocampal EEG activity was recorded by 7P511 amplifiers on a Grass Model 78 polygraph. Half-amplitude filters for pen writeout were set at 1 and 60; occasional use was made of the 60-cycle filter. In addition, two Krohn-Hite band pass filters (330-BR) were used to analyze EEG activity in various frequency ranges.

Following surgery, animals were allowed at least 1 week to recover. They were maintained throughout the experiment on food and water ad lib, except for occasional tests with $24-\mathrm{h}$ food deprivation. Consummatory tests (with bars retracted) were made with dishes of wet mash and water. A wooden block was also included to assess gnawing behavior. All stimulation in these tests was delivered on a noncontingent basis.

\section{RESULTS}

Figure 1 illustrates results obtained from one of the animals at threshold intensities of stimulation for eliciting consummatory behavior. First, it may be noted (Record A), in agreement with other findings, that normal exploratory behavior in the rat is associated with synchronous hippocampal EEG activity at a frequency of about $8 \mathrm{~Hz}$ and that normal feeding behavior induced by deprivation of food is associated with irregular or relatively desynchronized hippocampal activity (Record B). As shown in the accompanying 

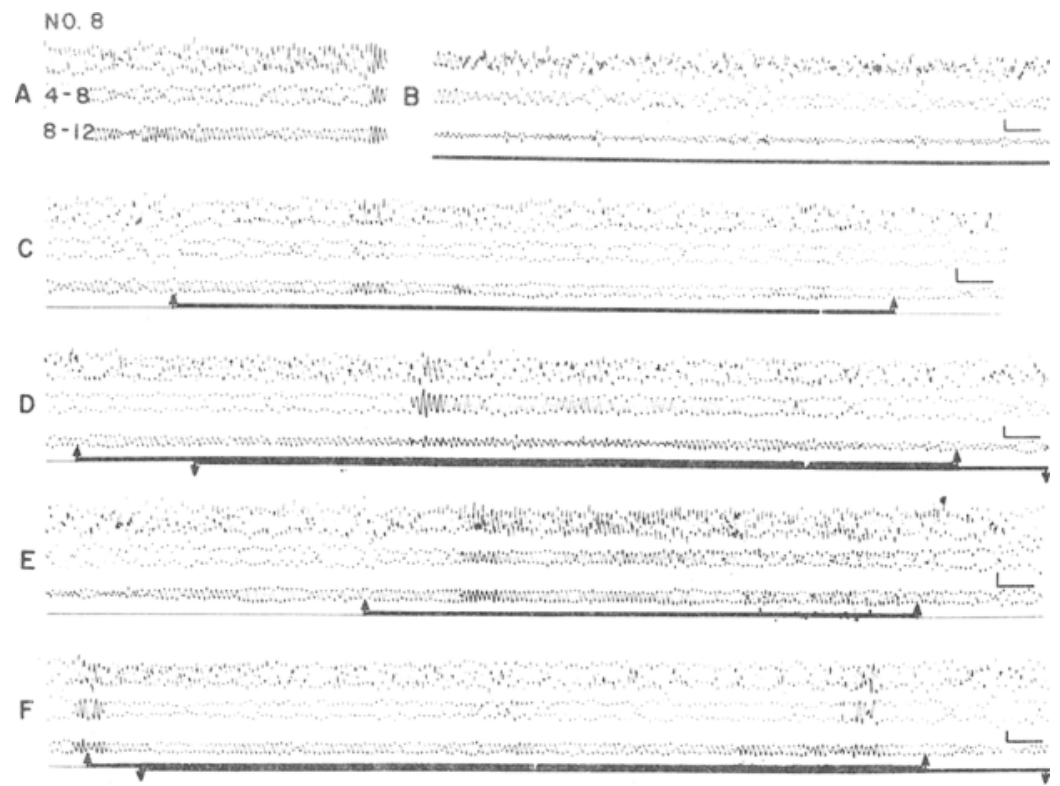

Fig. 1. Hippocampal electrical activity under different behavioral conditions. Record A: exploratory behavior. Record B: normal feeding behavior following 24-h food-deprivation. Record C: 50 microamperes hypothalamic stimulation (between upward arrows). Record D: 50 microamperes stimulus-bound eating (stimulation between upward arrows; eating between downward arrows). Record E: 65 microamperes stimulation. Record F: 65 microamperes stimulus-bound eating. In all cases the hippocampal site of recording was ipsilateral to the hypothalamic electrode. In each record the top trace is "unfiltered"; the bottom two traces show filtered recordings with frequency bands as indicated. Calibration: $1 \mathrm{sec}$ and 100 microvolts.

frequency analyses, the shift from searching to feeding behavior sharply attenuated EEG frequencies in the 8-12 range and reduced activity in the 4-8 range. However, it should be noted that this "blocking" of theta activity during consummatory behavior was never associated with changes in high-frequency hippocampal activity in the range of $20-40 \mathrm{~Hz}$.

As illustrated in Record C of Fig. 1, noncontingent threshold-intensity $\mathrm{LH}$ stimulation in the absence of relevant goal objects evoked searching behavior that was associated with synchronization of hippocampal activity at a frequency of 8-9 Hz. This synchronization persisted with varying amplitude and with slight variation in frequency throughout the train of stimulation, as did the evoked behavioral pattern. There was never any evidence of "rebound" desychronization at the offset of stimulation. Often, the theta pattern and active behavior continued for a period following the end of stimulation.

During stimulation-evoked eating behavior (Record D, Fig. 1), hippocampal theta activity persisted at $1.2 \mathrm{~Hz}$ below that observed during stimulation in the absence of feeding. The evoked feeding behavior blocked theta activity in the 8-12 band-pass recording (compare Records $\mathrm{C}$
Increased intensity of stimulation a frequency of $9-10 \mathrm{~Hz}$ during more active and B). Nevertheless, the evoked feeding was still associated with high-amplitude theta of $7-8 \mathrm{~Hz}$, as evident in the $4-8$ Records B and D). The evoked feeding also appeared to be somewhat more "agitated" and sometimes continued for a brief period after offset of the stimulation.

Increased intensity of stimulation in the during normal feeding behavior (com exploratory behavior (Record D). Note the decrease in amplitude of aciivity in the 4-8 band-pass recording. As shown in Record F, such stimulation also increased theta activity in the 8-12 range during evoked feeding behavior, although, again, the peak frequency was $1-2 \mathrm{~Hz}$ below that observed in the absence of food.

Extension of these tests to higher intensities of stimulation (Fig. 2) did not alter the pattern of results. In the absence of food (Record A), the more intense stimulation evoked more vigorous exploratory behavior and a peak theta frequency of about $10 \mathrm{~Hz}$. During stimulus-bound eating (Record B), the peak theta frequency reached $9 \mathrm{~Hz}$. As illustrated in Record C, there were no apparent changes in high-frequency hippocampal activity in the range of $20-40 \mathrm{~Hz}$ during either stimulus-evoked exploratory or consummatory behavior.

It may also be noted that $\mathrm{S} 8$ displayed strong self-stimulation for short 0.5 -sec trains at a minimum intensity of 70 microamperes. But this value should not be regarded as the "reward threshold" in any comparison with thresholds for evoked behavior, since with longer trains, as used in the recording tests, less intense stimulation can effectively reinforce responding.

The results obtained with the two other animals verified in all respects the findings illustrated for $S 8$. They also revealed similarity of evoked patterns in the dorsal hippocampus ipsilateral and contralateral to the site of hypothalamic stimulation. One animal also displayed stimulus-bound drinking. As with evoked eating, synchronization of hippocampal activity persisted during the consummatory behavior, with the frequency of theta attenuated by $1.2 \mathrm{~Hz}$. Increases in intensity of stimulation again served to augment the
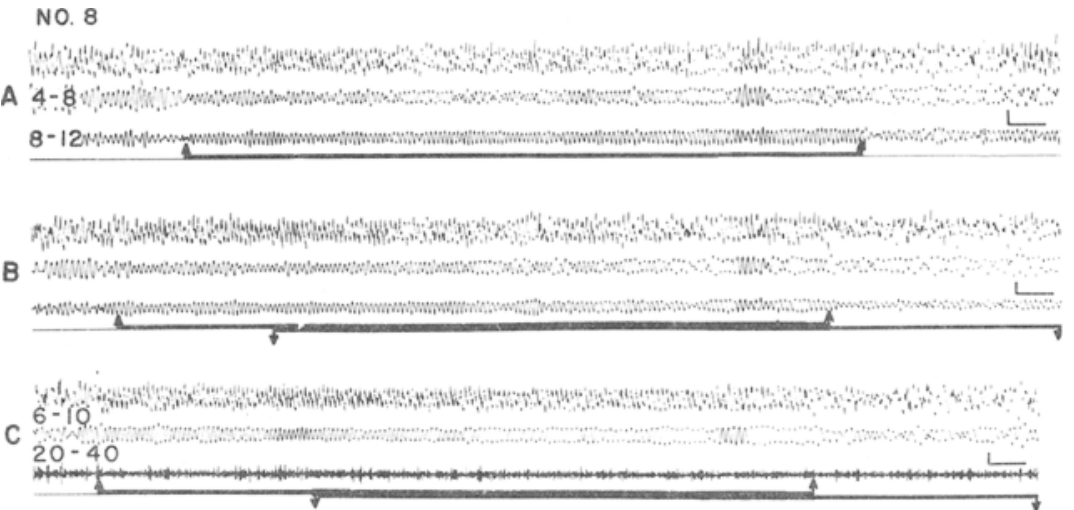

Fig. 2. Record A: hippocamp:" activity with 80 microamperes hypothalamic stimulation. Records $B$ and $C: 80$ microamperes stimulus-bound eating. Details are the same as in Fig. 1. Calibration: $1 \mathrm{sec}$ and 100 microvolts. 
pattern of results observed with threshold intensities.

Histological analysis indicated that the hypothalamic electrode in all three animals was located in the MFB slightly dorsolateral to the fornix. The dorsal hippocampal electrodes were located adjacent to pyramidal field CA3. DISCUSSION

Two major findings emerge from the present study. First, unlike normal eating and drinking, which are associated with irregular or desynchronized hippocampal activity, stimulus-bound consummatory behavior is associated basically with persistence of synchronized hippocampal activity. This difference probably does not reflect variation in the intensity of the disposition to eat or drink. Rather it suggests some differences in the electrophysiological state produced by the two sets of conditions. In stimulus-evoked behavior appetitive mechanisms may continue to operate during consummatory responses unlike the normal sequencing of appetitive and consummatory phases. It is also possible that negative properties of the stimulation, as evidenced by offset responses in tests of preferred duration (Pond \& Schwartzbaum, 1969), persist and contribute to the unusual agitated quality of stimulus-bound consummatory behavior.

At the same time, the results also indicate that stimulus-bound consummatory behavior can, with in limits, attenuate the frequency of evoked theta patterns. This effect may relate to a reduction in feedback from locomotor movement that is otherwise elicited-paralysis with d-tubocurarine, e.g., produces similar effects (Pond \& Schwartzbaum, 1970; Routtenberg \& Kramis, 1968)-although consummatory activity may operate in other ways to produce "deactivating" effects on nonspecific systems that may counteract properties of LH stimulation. Evidence of such deactivating effects is available from work with cats (Marczynski \& Hackett, 1969), and we have observed changes in sensory evoked response in rats as they switched from operant to consummatory behavior that are consistent with such a supposition. In any case, the attenuation of evoked theta frequency may signify some degree of change in appetitive state and/or negative properties of brain stimulation which may relate to increased preferred duration of brain stimulation observed during evoked consummatory-type behavior (Mendelson, 1969).

Finally, it is worth noting that the present data cast doubt on the generality of recent findings (Paxinos \& Bindra, 1970) that conditioned immobility to rewarding brain stimulation can block hippocampal theta otherwise evoked by the stimulation. It is likely that behavioral patterns expressed during stimulation can only alter evoked theta rhythms within limits. At higher intensities of stimulation, theta patterns may be attenuated but not blocked. Stimulus-evoked theta is not simply a function of the feedback from the evoked behavior.

\section{REFERENCES}

CHRISTOPHER, M., \& BUTTER, C. M. Consummatory behaviors and locomotor exploration evoked from self-stimulation sites in rats. Journal of Comparative \& Phy siological Psychology, 1968, 66, 335-339.

GRASTYAN, E., KARMOS, G., VARECZKEY, L., \& KELLENYI, L. The hippocampal electrical correlates of the homcostatic regulation of motivation. Electroencephalography \& Clinical Neurophysiology, 1966, 21, 34-53.

KRAMIS, R. C., \& ROUTTENBERG, A. Rewarding brain stimulation, hippocampal activity, and foot-stomping in the gerbil. Physiology \& Behavior, 1969, 4, 7-11.

MARCZYNSKI, T. J., \& HACKETT, J. T. Post-reinforcement electrocortical synchronization and facilitation of cortical somato-sensory evoked potentials during instrumentally conditioned appetitive behavior in the cat. Electroencephalography \& Clinical Neurophysiology, 1969, 26, 41-49.

MENDELSON, J. Lateral hypothalamic stimulation: Inhibition of aversive effects by feeding, drinking and gnawing. Science, 1969, $166,1431-1433$.

PAXINOS, G., \& BINDRA, D. Rewarding intracranial stimulation, movement and the hippocampal theta rhythm. Physiology \& Behavior, 1970, 5, 227-231.

POND, F. J., \& SCHWARTZBAUM, J. S. Preferred duration of rewarding brain stimulation in rats and interstimulus interval. Physiology \& Behavior, 1969, 4, 911-916.

POND, F. J., \& SCHWARTZBAUM, J. S. Hippocampal electrical activity evoked by rewarding and aversive brain stimulation in rats. Communications in Behavioral Biology, 1970 , in press.

ROUTTENBERG, A. Hippocampal correlates of consummatory and observed behavior. Physiology \& Behavior, 1968, 3, 533-535.

ROUTTENBERG, A., \& KRAMIS, R. C, Hippocampal correlates of aversive midbrain stimulation. Science, 1968, 160, 1363-1365. VALENSTEIN, E. S., COX, V. C., \& KAKOLEWSKI, J. W. The hypothalamus and motivated behavior. In J. T. Tapp (Ed.), Reinforcement and behavior. New York: Academic Press, 1969. Pp. 242-285.

VANDERWOLF, C. H. Hippocampal electrical activity and voluntary movement in the rat. Electroencephalography \& Clinical Neurophysiology, 1969, 26, 407-418.

\title{
Effects of ingesting nonnutritive sweet substances on subsequent sucrose consumption
}

\author{
ELVIS C. JONES \\ Frostburg State College, Frostburg, Md. 21532
}

An experiment with rats was conducted to verify and further study the drive-reducing effects of nonnutritive sweet substances. Ingestion of $1 \mathrm{ml}$ of $.25 \%$ pure saccharin or $.6 \%$ sodium cyclamate solutions significantly suppressed subsequent consumption of a $16 \%$ sucrose solution. The data also indicated that the suppression effect following consumption of saccharin disappears within $5 \mathrm{~min}$. It was concluded that the transitory effects of saccharin consumption may account for discrepancies between previous studies on the drive-reducing effects of saccharin.

Miller, Murray, \& Roberts (1955), found that sodium saccharin suppresses subsequent food consumption if the saccharin is taken orally, but not when introduced directly into the stomach. The suppression effect was attributed to oral-pharyngeal factors.

Important details of the Miller et al experiments are not generally available, and there has been little subsequent research directly related to this effect. The bearing this phenomenon has on theories of reinforcement is such that a general verification and extention seems worthwhile, especially since these findings are at odds with those reported by Hausmann (1933) and by Smith \& Duffy (1957). These investigators found that saccharin did not significantly decrease the amount of food ingested. The discrepancy between these findings may be due to the fact that the latter two experiments measured consumption over long periods. If the effect of saccharin is highly transitory, it may have been masked in these studies.

The present experiment represented an attempt to verify the apparent drive-reducing properties of saccharin (employing pure saccharin rather than 